\title{
ADDITIONAL NOTES ON THE GENUS VERBENA. III
}

\author{
Harold N. Moldenke
}

VERBENA [Dorst.] I.

Additional synonymy: Verbena [Tourn.] L. ex Migula, Exkursionsfl. Deutsch. 2: 134. 1906. Verbenia L. ex Moldenke, Alph. List Invalid Names Suppl. 1: 28, in syn. 1947; Runner, Rep. G. W. Groff Coll. 361. 1961.

Additional \& emended bibliography: Rumph., Herb. Amboin. 7: 60, pl. 27, fig. 2. 1755; Rohl., Deutschl. Fl., ed. 1, 2: 329 \& 553. 1812; Mossler, Gemein. Handb. Gew. 2: 816 \& Reg. 1815; W. D. J. Koch in ROhl., Deutschl. F1., ed. 2, 4: 211, 339, \& 744. 1833; W. D. J. Koch, Syn. F1. Germ., ed. I, 578 \& 844. 1837; Guill., Enum. P1. Déc. Voy. Iles Société 42 \& 209. 1837; W. D. J. Koch, Syn. Deutsch. \& Schw. F1., ed. 1, 575--576 \& Reg. 99. 1838; Meigen, Deutschl. FI. 3: 130 \& 620. 1842; W. D. J. Koch, Taschenb. Deutsch. Fl., ed. I, 477 \& 604. 1843; W. D. J. Koch, Syn. FI. Germ., ed. 2, 2: 664 \& 964 (1844) and ed. 2, 3: 1160. 1845; W. D. J. Koch, Syn. Deutsch. \& Schw. F1., ed. 2, 681-682 \& 1206. 1846; G. \& F. Lorinser, Taschenb. F1. Deutsch., ed. 1, 311 \& 488 (1847) and ed. 2, 311 \& 488. 1851; W. D. J. Koch, Taschenb. Deutsch. F1., ed. 3, 417 \& 604 (1851) and ed. 4, xliil, 400, \& 583. 1856; Langmann, F1. Nord\& Nitteldeutsch., ed. 2, $110,252-253$, \& 462. 1856; Guillard, Bull. Soc. Bot. France L: 122. 1857; W. D. J. Koch, Taschenb. Deutsch. F1., ed. 5, xli1i, 400, \& 583. 1860; Bocq., Adansonia 2: $86,106-107,110-113,115,118,123,124,126-128,130-133$, $136,139,140,142,144,146,147,149,152-154,157$, \& 158 (1862) and 3: $178,180,181,183,185$, \& 201-204. 1863; Bocq., Rev. Verbenac. 86, 106-107, 110-113, 115, 118, 123, 124, 126$128,130-133,136,139,140,142,144,146,147,149,152-154$, $157,158,178,180,181,183,185$, \& $201-204$. 1863; W. D. J. Koch, Taschenb. Deutsch. F1., ed. 6, xli11, 400, \& 583. 1865; Langethal, Beschreib. Gew. Deutsch., ed.'2, 448 \& 786. 1868; Niq., Cat. Mus . Bot. Lugd.-Bat. 70. 1870; E. Hall. in W. D. J. Koch, Taschenb. Deutsch. Fl., ed. 7, 403 \& 802 (1878) and ed. 8, 403 \& 802. 1881; Lefroy, Bull. U. S. Nat. Mus. 25: 96. 1884; K. G. Lutz, Pflanzenfr. 93 \& 128, pl. 17, fig. 19. 1887; W. Kedic., Illustr. Pflanzenb. vili, xocix, \& 1i5, pl. 50, fig. 112 \& 112a. 1893; C. E. Bessey, Essent. Bot., ed. 7, 287, 334, \& 355. 1896; J. Matsum., Bot. Mag . Tokyo 13: [i13]-1ن. 1899; Ichimura, Bot. Hag. Tokgo 13: 116. 1899; Migula, Exkursionsfl. Deutsch. 2: 134 \& 185. 1906; Hall. \& Brand in $\mathrm{W}$. D. J. Koch, Syn. Deutsch. FI., ed. 3, 3: 2156 \& 3088. 1907; Kawakamí, List P1. Formos. 85. 1910; W. Stone, Ann. Rep. N. J. State Mus. 1910: 660-661 \& 827. 1911; A. Stewart, Proc. Calif. Acad. Sci., ser. 4, 1: 134. 1911; Dunn \& Tutcher, Ken Bull. Hisc. Inf. Addit. Ser. 10: 201 \& 202. 1912; E. D. Yerr., Interpret. Rumph. Herb. Amboin. 547. 1917; C. Robertson, Psyche 29: 159-173. 1922; Gamble, FI. Madras 1085 \& 1106.1924 ; S. Sasaki, List PI. 
Formos. 353. 1928; H. W. Harvey, Ga. State Coll. Agr. Ext. Div. Bull. 402, ed. 1, 34 \& [43]. 1931; Hand.-Mazz., Ann. Hort. Gothenb. 9: [67] . 1934; Sampaio, Bol. Mus. Nac. Rio Jan. 13: 190, 191, 234, 240, \& 292. 1937; R. W. R. Villl., Gard. Book Barbados 74 \& x. 1935; Fletcher, Kew Bull. Misc. Inf. 1938: 408 \& 410--411. 1938; Hottes, Book Shrubs, ed. 4, 404. 1942; Blakelock, Kew Bull. Misc. Inf. 1949: 539. 1949; Brenan, Kew Bull. Misc. Inf. 1950: 224 \& 226. 1950; Chittenden, Roy. Hort. Soc. Dict. Gard. 3: 1188 \& 1189 (1951) and 4: 1898. 1951; Bodger, Electros Fls. 27, 61, 95, \& 105, pl. 54g, 4 lile, 573c, 956a, 1137f, \& 3018f. 1952; K. H. Rechinger, Arkiv Bot., ser. 2, 2: 408. 1952; H. C. Greene, Trans. Wisc. Acad. Sci. 42: 70. 1953; Bor \& Raizada, Some Beaut. Ind. Climbers [136] . 1954; Berhaut, FI. Sénegal 65. 1954; Darlington \& Wylie, Chromosome AtI., pr. 1, 322-323 \& 518. 1955; E. W. Lathrop, Univ. Kans. Sci. Bull. 39: 124, 125, 137, 172, \& 173. 1958; Mostert, Bot. Surv. S. Afr. Mem. 31: 41 \& 191. 1958; T. A. Rao, Bull. Bot. Surv. Ind. 1: 114. 1959; K. H. Rechinger, Arkiv Bot., ser. 2, 5: 346. 1959; J. Hutchinson, Fam. Flow. PI., ed. 2, 2: 395. 1959; Anon., Kew Bull. Gen. Index 1929-1956, 291. 1959; Е. H. Moss, F1. Alberta 397. 1959; Nath, Bot. Surv. South. Shan States 143. 1960; Gupta \& Marlange, Trav. Sect. Scient. Inst. Franç. Pond. 3 (1): 4 \& 11. 1961; Thomen, AtI. Poche FI. Suisse 2198. 1961; Runner, Rep. G. W. Groff Coll. 361. 1961; Darlington \& Wylie, Chromosome Atl., pr. 2, 322--323 \& 518. 1961; Nair \& Rehman, Bull. Nat. Bot. Gard. Lucknow $76: 3-4$ \& 8. 1962; Clapham, Tutin, \& Warburg, FI. Brit. Isles, ed. 2, 729-730. 1962; Lid, Norsk \& Svensk. F1. 568 \& 569, fig. 34la. 1963; Quezel \& Santa, Nouv. Fl. Algérie 2: 779 \& 780.'1963; Huber, Heppel, \& Meikle in Hutchinson \& Dalz., FI. W. Trop. Afr., ed. 2, 2: 432. 1963; Hepper in Hutchinson \& Dalz., FI. W. Trop. Afr., ed. 2, 2: 434. 1963; Meikle in Hutchinson \& Dalz., F1. W. Trop. Afr., ed. 2, 2: 437. 1963; Balakrishnan, Bull. Bot. Surv. Ind. 6: 81--92. 1964; H. C. Greene, Trans. Wisc. Acad. Sc1. 53: 194-195. 1964; Ungar, Univ. Kans. Sci. Bull. 46: 18, 19, \& 28. 1965; Percival, Flor. Biol. 131-132. 1965; Backer \& Bakh., F1. Java 2: 595 \& 596. 1965; J. E. Weaver, Native Veg. Nebr. 25.1965; A. D. Hawkes, Guide P1. Everglades Nat. Park 43 \& [51] 1965; Liogier, Rhodora 67: 349. 1965; Solbrig, Castanea 30: 173-174. 1966; Rickett, Wild Fls. U.S. I (2): 361--364, pl. 112 \& 113. 1966; C. B. Clarkson, Castanea 31: 100. 1966; Balakrishnan, Biol. Abstr. 47: 4615. 1966; T. Swain, Compar. Phytochem. 348. 1966; Bate-Smith \& Swain in T. Swain, Compar. Phytochem. 166 \& 170. 1966; Harborne in T. Swain, Compar. Phytochem. 285. 1966; Moldenke, Phytologia 13: 243-276, 307, 366, 401, 509, \& 512 (1966) and 14: 175. 1966; G. Taylor, Ind. Kew. Suppl. 13: 143. 1966; Anon., Gen. Costa Ric. Phan. 10.1966; Moldenke, Resume Suppl. I4: 1-3, 5, 9, \& 10. 1966; Anon., Assoc. Etud. Tax. F1. Afr. Trop. Index 1965: 67. 1966; Kitamura, Result Ky Kyoto Univ. Scient. Exped. Karakoram 8: 132. 1966; J. Gomez, Biota 6: 103, 107, 109, 114-116, \& 119. 1966; R. C. Jacks., Reg. Veget. 43: 59. 1966; Stearn, Botan. Latin 295. 1966; Anon., Biol. Anstr. 47 (20): S.67 \& S.162. 1966; Herbst Bros., Seeds for. Nurserymen 23. 1966; G. B. Foster, Herbs for Every Gard. 199. 1966; Ross-Craig, Drawings Brit. P1. 23: pl. 38. 1966. 
Robertson (1922) and Percival (1965) report that Verbenajus verbenae is an oligolectis bee of the family Panurgidae which collects solely from three species of Verbena, viz., V. hastata L., V. stricta Vent., and V. urticifolia L. Mostert (1958) reports that an unidentified prostrate herbaceous Verbena species was recorded from Bloemfontein, South Africa, represented by G. Potts 2079. Weaver (1965) mentions but does not identify a mwoods vervain" found in Nebraska only in woodlands, not on the prairies.

The Heddle 2816, distributed as a species of Verbena, is actually Lantana macropoda Torr., while M. C. Johnston 2664, Waterfall \& Wallis 13476, and Weintraub \& Roller 173 are Priva grandiflora (Ort.) Moldenke, and Bowman s.n. [Sept. $29,195 \overline{7 \text { ] is Salvia occi- }}$ dentalis $\mathrm{Sw}$. in the Lamiaceae.

VERBENA ALATA Sweet

Additional bibliography: Bocq., Adansonia 3: [Rev. Verbenac.] 202. 1863; Moldenke, Phytologia 13: 181. 1966.

VERBENA AMBROSIFOLIA Rydb.

Additional bibliography: Darlington \& Wylie, Chromosome Atl., pr. I, 323 (1955) and pr. 2, 323. 1961; Moldenke, Phytologia 13: 244. 1966.

Additional citations: TEXAS: Brewster Co.: D. S. Correll 33820 (Id).

VERBENA BERTERII (Meisn.) Schau.

Additional bibliography: Bocq., Adansonia 2: 126 (1862) and 3: 202. 1863; Bocq., Rev. Verbenac. I26 \& 202. 1863; Moldenke, Phytologia 13: 183. 1966.

VERBENAA BIPINNATIFIDA Nutt.

Emended synonymy: Verbena bipinnatifida Schau. apud Bocq., Adansonia 3: 202. 1863.

Additional bibliography: Bocq., Adansonia 3: [Rev. Verbenac.] 202. 1863; Moldenke, Phytologia 13: 244--246. 1966; Rickett, Wild Fls. U. S. I (2): 364.1966.

Jones found this species growing in post oak savannas.

Additional citations: TEXAS: Bexar Co.: Martz \& Martz s.n. [June 3, 1959] (Ws). Brom Co.: Benson \& MeCart 9334 (Id); Eming 27 (Ld). Burnet Co.: J. Jones I (Id); F. Sylvester 2 (Id). Coloman Co.: J. Folkner 13 (Ld); H. Spoon 13 (Ld). Coryell Co.: S. Jackson 그 (Id). Eastland Co.: Wheeless 18 (Ld). Foard Co.: C. Turner 17 (Id). Garza Co.: B. Jensen 8 (Id). Kaufman Co.: C. Duke 10 (Id). San Saba Co.: CaIhoun 13 (Id); E. Howell I (Id). Williamson Co.: J. Sargent 6 (Id).

XVERBENA BLANCHARDI Moldenke

Additional bibliography: E. W. Lathrop, Univ. Kans. Sci. Bull. 
39: 172. 1958; Moldenke, Phytologia 13: 184. 1966.

\section{VERBENA BONARIENSIS L.}

Additional bibliography: Guill., Enum. Pl. Dec. Voy. Iles Soc1été 209. 1837; Bocq., Adansonia 3: [Rev. Verbenac.] 202. 1863; Dunn \& Tutcher, Kew Buil. Misc. Inf. Addit. Ser. 10: 201. 1912; Gamble, Fl. Madras 1106. 1924; Sampaio, Bol. Mus. Nac. Rio Jan. 13: 191. 1937; Darlington \& Wylie, Chromosome Atl., pr. 1, 323. 1955; Mostert, Bot. Surv. S. Afr. Mem. 31: 41 \& 191. 1958; Darlington \& Wylie, Chromosome AtI., pr. 2, 323. 1961; Backer \& Bakh, F1. Java 2: 596. 1965; Liogier, Rhodora 67: 349. 1965; Koldenke, Phytologia 13: 245. 1966.

Recent collectors describe this plant as having the calyx green below to reddish above, the corolla whitish below, reddish in the central region, and lavender above, with white hairs, the anthers yellow-green, and the pistil green, flowering in June, and growing at 2500 to 4500 feet altitude in Jamaica. Backer \& Bakhuizen van den Brink (1965) report that in Java it grows in agricultural fields, Cinchons plantations, grassfields, and along roadsides. Mostert (1958) states that in South Africa it is found on streambanks, dams, pans, flooded and irrigated areas subject to wetness and drought, severe grazing, and trampling, while Sidey refers to it there as a "shrub 5-6 feet tall", with purple flowers. Sampaio (1937) records the vernacular name "cambark de capoera" .

Additional citations: JAMAICA: Crosby, Hespenheide, \& Anderson 231 (Wi); Yuncker 18674 (Mi). SOUTH AFRICA: Cape of Good Hope: Sidey $3772(\mathrm{~S})$.

VERBENA BRACTEATA Lag. \& Rodr.

Additional bibliography: Bocq., Adansonia 3: [Rev. Verbenac.] 202. 1863; E. W. Lathrop, Univ. Kans. Sc1. Bull. 39: 173. 1958; E. H. Hoss, F1. Alberta 397. 1959; Ungar, Univ. Kans. Sci. Bull. 46: 28. 1965; Rickett, wild Fls. U. S. 1 (2): 362 , pl. 112. 1966; R. B. Clarkson, Castanea 31: 100. 1966; Moldenke, Phytologia 13: 245.1966.

Additional illustrations: Rickett, Wild Fls. U.S. 1 (2): pl. 112 [in color]. 1966.

Holmgren \& Reveal 1023 is said to have had mblue-violet" corollas and was "infrequent" in wet gravelly soil along the levee of a ditch at 6300 feet altitude in Nevada, flowering in June. Moss (1959) calls this plant "carpet vervain" and describes it as having branches more or less hirsute and blue to purple corollas, growing on "stream flats, roadsides and waste places in the prairie region" of Alberta. Lathrop (1958) describes the habitat of the species as "fields, waste places, and pastures", while Ungar (1965) tells us that it is "prominent" on grazed sandhills in the therophyte formation.

Additional citations: NEVADA: White Pine Co.: Holmgren \& Reveal 1023 (Mi). NEBRASKA: Buffalo Co.: Guthe s.n. [Kearney, Juñe 1893] (III). TEXAS: Floyd Co.: Purvis $27 \frac{1}{(\mathrm{Id})}$ 
VERBENA BRASIIIENSIS VeIl.

Additional synonymy: Verbena brasiliensis L., in herb.

Additional bibliography: Sampaio, Bol. Ius. Nac. Rio Jan. 13: 240. 1937; Moldenke, Phytologia 13: 245. 1966; Rickett, Wild Fls. U. S. 1 (2): 364 . 1966.

Recent collectors describe this plant as 2-3 feet tall, with a minty odor, growing at 10 w as 200 meters altitude in Peru. Fiten found it in Săo Paulo growing in waste ground in the shade of a large leguminous tree, in poor soil - reddish-brown fine sand with a little clay - in what was originally cerrado vegetation; Ugent found it on sunny lichen-covered rock piles among Inca ruins in Cuzco; while Iltis and his associates found it among weeds along roadsides and in highway center-strips, in cultivated potato fields, and among weeds in fertile deep-soil cabbage (Brassica oleraces) fields along with abundant Solanum calcense and also Rumex, Fragrostis, 0xalis, Polygomum, Galinsoga, Tagetes, etc. The flowers on G. Biten 1595 are described as having been "light-purple".

Additional citations: PFRU: Cuzco: Iltis, Iltis, Ugent, \& Ugent 891 (Ws); D. Ugent 5354 (Rf). Junin: Iltis, Il tis, Ugent, \& Ugent $347(\mathrm{Ac})$. Iima: Iltis, Iltis, Ugent, \& Ugent 390 (Rf). BRAZIL: Săo Panlo: G. E1ten 1595 (N).

VERBENA CAURRONENSIS I. I. Davis 1966.

Additional bibliography: Moldenke, Phytologia 13: 24,5 \& 257 .

VERBBENA CANAADENSIS (L.) Britton

Additional bibliography: Bocq., Adansonta 2: 126 (1862) and 3: 202. 1863; Bocq., Rev. Verbenac. 126 \& 202. 1863; Darlington \& Wylie, Chromosome AtI., pr. 1, 323. 1955; E. W. Lathrop, Univ. Kans.'Sc1. Bull. 39: 125 \& 173. 1958; Darlington \& Wylie, Chromosome Atl., pr. 2, 323. 1961; Moldenke, Phytologia 13: 245-2l,6. 1966; Rickett, Wild Fls. U. S. I (2); 362 \& 364, pl. 112.1966.

Additional illustrations: Rickett, wild Fls. U. S. I (2): pl. 112 [in color] 1966.

Lathrop (1958) telis us that this plant is frequent and prominent on prairies in the spring, in rocky prairie pastures and prairie woodlands, in open woods, and along roadsides.

Additional citations: TEXAS: Henderson Co.: Correll \& Correll 33960 (Id). County undetermined: T. Drummond $263(\mathrm{~S})$.

VERBBENA CANESCENS H.B.K.

Additional bibliography: Bocq., Adansonta 3: [Rev. Verbenac.] 202. 1863; Moldenke, Phytologia 13: 2l,6. 1966.

Recent collectors have found this plant growing on dry grazed slopes with Agave, Opuntia, Acacia, and Solanum polyadenium and on grazed roadsides and in drainage ditches with Yucca, Bouvardia, and cacti, in mesquite grasslands now becoming desert. The flowers are described as mblue" on Roe, Roe, \& Yori 4 . 
Additional citations: MEXICO: Coahuila: Roe, Roe, \& Mori 4 (Ws). Puebla: Ugent \& Flores C. 2539 (Ws).

VERBENA GANESCENS var. ROEMERIANA (Scheele) Perry

Additional bibliography: Moldenke, Phytologia 13: 246. 1966.

Runyon describes this plant as a low annual herb with many branches above the base, frequent on open clay soil, and with blue flowers.

Additional citations: TEXAS: Brown Co.: Benson \& McCart 9335 (Id). Cameron Co.: R. Runyon 6067 (Ld). Coleman Co.: B. E. Holland 2l (Ld). Travis Co.: Webster \& Webster 115 (Mi).

VERBENA CAROLINA I.

Additional bibliography: Bocq., Adansonia 3: [Rev. Verbenac.] 203. 1863; A. Stewart, Proc. Calif. Acad. Sc1., ser. 4, 1: 134. 1911; Moldenke, Phytologia 13: 246, 251, \& 258. 1966; Koldenke, Résumé Suppl. 14: 2. 1966.

Breedlove found this species growing on grassy slopes, grassy road banks, and in large moist pastures. He describes the flowers as "blue" on his nos. 10457 \& 11452 and as "pale-blue" on no. 10519. Feddema 1659 is said to have had the "corolla white with lavender ring in throat", and is said to have been "an occasional weed at field margins" in weedy fallow cornfields.

Other recent collectors have found the plant growing in grazed fire-scarred pine woods, growing through a thick layer of pine needles mulch, along very moist grazed roadsides, and in brush piles, as well as in open spots in dense Pinus and Quercus woods along with Solanum skutchii and S. stenophyllidium. The flowers of Roe, Roe, \& Mori 1530 are said to have been "lavender". The R. I. Bowman 8I, distributed as V. carolina, is actually V. sedula Mol denke.

Additional citations: MEXICO: Chiapas: Breedlove 10457 (Ac), 10519 (Rf). Guerrero: Feddema 1659 (Mi). México: Roe, Roe, \& Mori 1530 (Rf). Michoacán: Ugent \& Flores C. 2217 (Ws), 2245

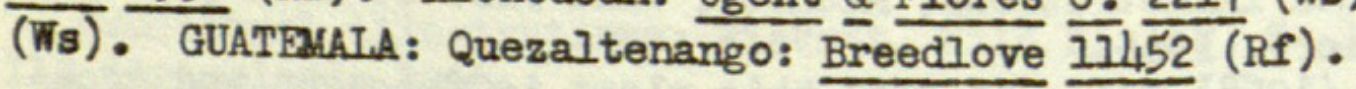

VERBENA CILIATA Benth.

Additional bibliography: Moldenke, Phytologia 13: 246, 257, \& 273. 1966; Moldenke, Résumé Suppl. I1: 1 \& 10. 1966.

Feddema describes this plant as an "occasional weed of cornfields" in Jalisco. His no. 2335 is said to have had "blue-lavender" flowers. The flowers on Mick \& Roe 15 are said to have been "violet" in color, but those on Roe, Roe, \& Kori 10, 23, \& 40 were

Recent collectors have found this plant growing along grazed roadsides and drainage ditches with Yucca, Bouvardia, and cacti in mesquite grasslands now becoming desert, on heavily grazed roadsides with species of Yucca, Acacia, and cacti in highly disturbed mesquite grassland vegetation with cornfields, and on dry grazed 
slopes with Agave, Opuntia, Acacia, and Solanum polyadenium. Mick \& Roe call the species "common" in deserts with "extensive desert pavement", along with Agave, Aloz, and cacti.

Additional citations: MEXICO: Coahuila: Mick \& Roe 15 (Rf); Roe, Roe, \& Mori 10 (Ws), 23 (Ac). Jalisco: Feddema 2335 (Mi). Nuevo León: Roe, Roe, \& Mori 40 (Ws). Puebla: Ugent \& Flores C. 2522 (Ws).

VERBENA CILIATA var. LONGIDENTATA Perry

Additional bibliography: Woldenke, Phytologia 13: 246 \& 247 . 1966; Holdenke, Résumé Suppl. 11: 10. 1966.

VERBEANA CILIATA var. PUBERA (Greene) Perry

Additional bibliography: Moldenke, Phytologia 13: 246, 247, \& 257. 1966; Koldenke, Résumé Suppl. 14: 1. 1966.

VERBENA CLAVATA Rufz \& Pav.

Additional bibliography: Bocq., Adansonia 2: 126. 1862; Bocq., Rev. Verbenac. 126. 1863; Moldenke, Phytologia 13: 190. 1966.

VERBENA COCHABAMBENSIS Moldenke

Additional bibliography: Moldenke, Phytologia 10: 103. 1964.

Recent collectors have found this plant growing on dry rocky bluffs above roadsides, flowering and fruiting in Nay.

Additional citations: BOITVIA: Cochabamba: Ugent \& Ugent 5223 (Ac, $\mathrm{Rf}$ ).

VERBENA CORYMBOSA Rúz \& Pav.

Additional bibliography: Bocq., Adansonia 3: [Rev. Verbenac.] 202. 1863; Darlington \& Wylie, Chromosome Atl., pr. 1, 323 (1955) and pr. 2, 323. 1961; Moldenke, Phytologia 13: 247. 1966.

VERBENA CRITHMIFOLIA Gill. \& Hook.

Additional bibliography: Darlington \& Wylie, Chromosome Atl., pr. 1, 323 (1955) and pr. 2, 323. 1961; Loldenke, Phytologia 11: 454. 1965 .

VERBENA CUNEIFOIIA RuIZ \& Pav.

Additional bibliography: Bocq., Adansonia 3: [Rev. Verbenac.] 203. 1863; Moldenke, Phytologia 13: 191. 1966; J. Gomez, Biota 6: $107,109,115$, \& 119. 1966.

Gómez (1966) records for this species the vernacular name "paralso", a name also applied to $V_{\text {. }}$ fasciculata Benth.

VERBEANA DELTICOIA SmaII 1966.

Additional bibliography: Moldenke, Phytologia 13: 247 \& 274 .

VERBENA DISSECTA Willd.

Additional bibliography: Bocq., Adansonia 3: [Rev, Verbenac.] 203. 1863; Darlington \& Wylie, Chromosome Atl., pr. 1, 322 (1955) 
and pr. 2, 322. 1961; Koldenke, Phytologia 13: 247-248, 251, 255, $256,262,263,268,270$, \& 271. 1966.

Bocquillon" (1863) refers to this taxon as "V. dissecta Walp.", a binomial referred by me to V. sulphurea D. Don.

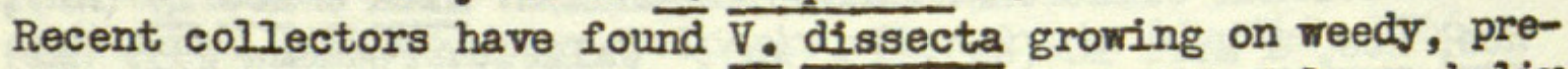
viously cultivated, but now fallow slopes along with Solamu boliviense, S. radicans, Chenopodium, Tagetes, Setaria, and Plantago.

Additional citations: BOLIVIA: Chuquisaca: Ugent \& Cárdenas 4988 (Ac, Rf).

VERBENA DISSECTA $f$. ALBA Moldenke 1966.

Additional bibliography: Moldenke, Phytologia 13: 24,8 \& 255.

VERBENA ELEGANS H.B.K.

Additional bibliography: Moldenke, Phytologia 13: 248. 1966; Moldenke, Résumé Suppl. 14: 2. 1966.

Recent collectors have encountered this plant on steep slopes with Quercus and on east-facing limestone cliffs with Acacia and Agave in Quercus scrub vegetation on rolling hills in the mesquitegrassland zone. The flowers are described as "purple" on Breedlove 12421 and as "maroon-red" on Roe, Roe, \& Mori 123.

Additional citations: MEXICO: Chiapas: Breedlove 12421 (Ac). San Iuis Potosi: Roe, Roe, \& Mori 123 (Ac).

XVERBENA ENGETMANNII Moldenke

Additional bibliography: Yoldenke, Phytologia 13: 248. 1966; Moldenke, Résumé Suppl. I1: 1. 1966.

One of the A. R. Moldenke 823 specimens previously cited as deposited in my personal herbarium is now in that of the Texas Research Foundation at Renner, Texas.

Additional citations: NEW YORK: County undetermined: J. Torrey s.n. (Mi). ILITNOIS: Marion Co.: A. R. Moldenke $126 I$ (Rf). MISSOURI: Ralls Co.: A. R. Moldenke $1 \overline{278}$ (Ac). Randolph Co.: A. R. Koldenke $128 \mathrm{I}$ (Rf) - MOUNTED ILLUSTRATIONS: A. R. Moldenke Kod. $9 \mathrm{~b}$ (z), $22(\mathrm{z}), 23(\mathrm{z})$.

VERBERIA FASCICULATA Benth.

Emended synonymy: Verbena mathemsii Briq. ex Ioldenke, Phytologia 9: 115, in syn. 1963; J. Gomez, Biota 6: 103, 107, \& 119.1966. Additional bibliography: Koldenke, Phytologia 11: 457.1965 ; J. Gomez, Biota 6: 103, 107, \& 119. 1966.

Gbmez (1966) records the vernacular name "parafso" for this species, a name also applied to V. cuneifolia Rulz \& Pav.

xVERBEANA FECUNAA Moldenke

Additional bibliography: Yoldenke, Phytologia 13: 195. 1966; G. Taylor, Ind. Kew. Suppl. 13: 143. 1966. 
XVERBEANA FERAX Moldenke

Additional bibliography: Moldenke, Phytologia 13: 195. 1966; G. Taylor, Ind. Kew. Supp1. 13: 143.1966.

VERBENA FLAVA Gill. \& Hook.

Additional bibliography: Darlington \& Wylie, Chromosome Atl., pr. 1, 322 (1955) and pr. 2, 322. 1961; Moldenke, Phytologia 11: 458. 1965 .

VERBENA GLABRATA H.B.K.

Additional bibliography: Moldenke, Phytologia 11: 458. 1965.

The A. Stewart 3317, previously cited by me as $\nabla$. glabrata, is actually the type collection of V. glabrata var. temuispicata Moldenke.

VERBENA GLABRATA var. TENUISPICATA Moldenke, var. not.

Haec varietas a forma typica speciel spicis tenuissimis recedit.

This variety differs from the typical form of the species in having its inflorescences much branched, the peduncles and individual spikes being extrenely slender, the fruits sparse and not contiguous.

The type of this variety was collected by Alban Stewart (no. 3317) at Villamil, on Albemarle Island, Galapagos Islands, on August 23, 1905, and is deposited in the Britton Herbarium at the New York Botanical Garden. The collector notes that the plant is cormon from 600 to 1400 feet altitude and on the floor of the crater at 2750 feet. This taxon was included by me in typical V. glabrata H.B.K. in previous installments of these notes.

Citations: GALAPAGOS ISIAANDS: Albemarle: A. Stewart 3317 (Gg31375--isotype, N-type).

VERBENA GLUTINOSA Kuntze (Mi).

Additional bibliography: Moldenke, Phytologia 13: 196. 1966.

Additional citations: ARGENIINA: Mendoza: Ruf zeal 7665/14

XVERBENA GOODMANI Yoldenike

Additional bibliography: Moldenke, Phytologia 13: 197. 1966; G. Taylor, Ind. Kem. Suppl. 13: 143. 1966.

VERBEANA GRACILESCENS (Cham.) Herter

Additional bibliography: Darlington \& Wylie, Chromosome Atl., pr. 1, 323 (1955) and pr. 2, 323. 1961; Moldenke, Phytologia 13: 197. 1966.

VERBBENA GRISEA Robinson \& Greerm.

Additional bibliography: A. Stewart, Proc. Calif. Acad. Sci., ser. 4, 1: 134. 1911; Moldenke, Phytologia 9: 158. 1963.

The type of this rare species was collected by G. Bauer (no. 180) on Duncan Island, Galapagos Islands, in August, 1891, and is deposited in the Gray Herbarium of Harvard University at Cambridge, Nas- 
Vol. 14, no. 5

sachusetts.

Additional citations: GAJAPAGOS ISLANDS: Duncan: G. Bauer 180 (Du-photo of type).

VERBENA GYNOBASIS Wedd.

Additional bibliography: Bocq., Adansonia 3: [Rev. Verbenac.] 203. 1863; Moldenke, Phytologia 13: 197. 1966.

VERBENA HALEI SmaIl

Additional bibliography: Moldenke, Phytologia $13: 247-249$ \& 254. 1966; Moldenke, Résume Suppl. 14: 1. 1966.

Additional citations: TEXAS: Bell Co.: M. Latham 7 (Id). Brow Co.: Ewing 28 (Id); J. Wheeler 14 (Id). Eastland Co.: Theeless 17 (Id). Frio Co.: Drews 6 (Id). Kleberg Co.: J. R. Crutchfield $\overline{1088}$ (Id). Medina Co.: Cope 26 (Ld). San Saba Co.: I. J. Jones 18 (Id).

VERBENA HASSLERANA Briq.

Additional bibliography: Moldenke, Phytologia 13: 199. 1966; Stearn, Botan. Latin 295. 1966.

VERBENA HASTATA I.

Additional bibliography: Bocq., Adansonia 2: 112 (1862) and 3: 203. 1863; Bocq., Rev. Verbenac. 112 \& 203. 1863; W. Stone, Ann. Rep. N. J. State Mus. 1910: 660. 1911; C. Robertson, Psyche 29: 159-173. 1922; E. W. Lathrop, Univ. Kans. Sci. Buli. 39: 173. 1958; H. C. Greene, Trans. Wisc. Acad. Sc1. 53: 195. 1964; Ungar, Univ. Kans. Sci. Bull. 46: 18, 19, \& 28. 1965; Percival, Flor. Biol. 131. 1965; R. B. Clarkson, Castanea 31: 100. 1966; Moldenke, Phytologia 13: 249 \& 260. 1966; Rickett, Wild Fls. U. S. I (2): 362, pl. 112. 1966.

Additional illustrations: Rickett, Wild Fls. U. S. I (2): pl. 112 [in color]. 1966.

Robertson (1922) and Percival (1965) report that the oligolectic bee, Verbenajus verbenae, of the family Panurgidae, collects only from this species, V. stricta Vent., and V. urticifolia I.

Greene (1964) reports that $V_{0}$ hastata is host to the fungus Ascochyta cuneomaculata Greene. Lathrop found the plant growing in rocky prairie pastures in Kansas, while Ungar (1965) describes it, from the same state, as a prominent surmer-bloomer in Scirpus americams - Eleocharis rostellata communities, in the hemicryptophyte life formation on freshwater seeps, and among Distichlis stricta, Eustoma grandiflora, Asclepias incarnata, and Vernonia fasciculata as the most prominent flowering plants in the spartina pectinata communty during the summer season.

Additional citations: NEW HAMPSHIRE: Carroll Co.: A. R. Molden-

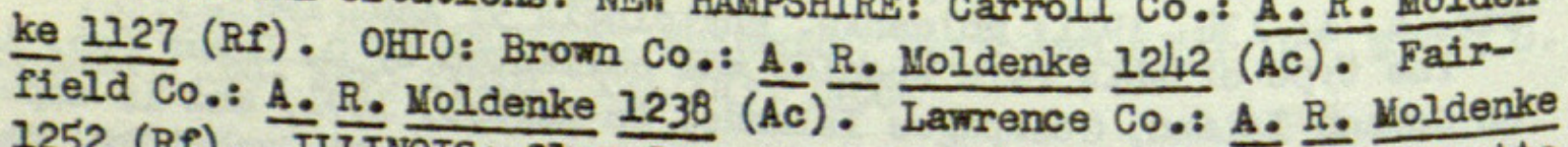

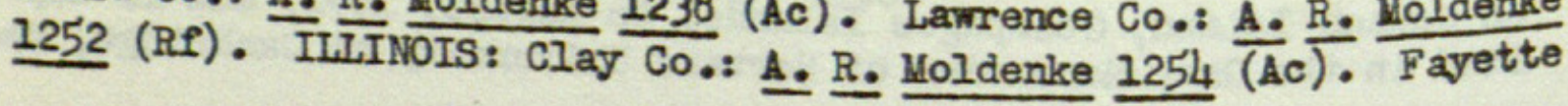


Co.: A. R. Moldenke 1263 (Rf). Macoupin Co.: A. R. Moldenke 1268 (Rf). Marion Co.: A. R. Moldenke 1258 (Ac). Montgomery Co.: A. R. Moldenke 1265 (AC). MINNESOTA: Ottertail Co.: P. Johnson $5 \overline{45}$ (Mi). KANSAS: Dickinson Co.: A. R. Moldenke 1297 (Rf). Osage Co.: A. R. Moldenke 1303 (Ac). MISSOURI: Monroe Co.: A. R. Mol-

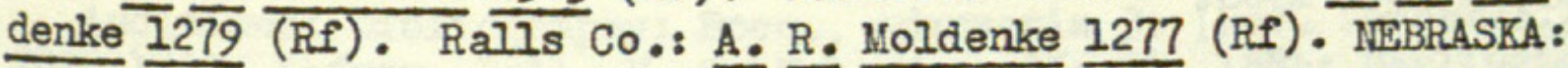
Buffalo Co.: Guthe s.n. [Kearney, Aug. 1893] (Mi). MOUNTED ILLUSTRATIONS: A. R. KoI denke Kod. $7 \mathrm{~b}(\mathrm{z}), 8 \mathrm{~b}(\mathrm{z}), 9 \mathrm{a}(\mathrm{z}), 1 \mathrm{lb}(\mathrm{z})$, $14 \mathrm{c}(\mathrm{z}), 1 \overline{\mathrm{C}}(\overline{\mathrm{z}}), \overline{17(\mathrm{z})}, \overline{20 \mathrm{~b}}(\mathrm{z}), 2 \mathrm{lb}(\mathrm{z})$.

VIERBENA HASTATA $f$. ROSEA Cheney

Additional bibliography: Moldenke, Phytologia 13: 200. 1966.

Additional citations: MISSOURI: Ralls Co.: A. R. Holdenke 1276

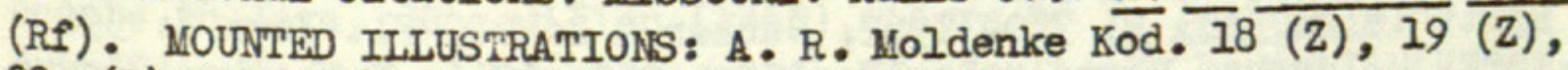
$20 \mathrm{a}(\mathrm{z}), 2 \mathrm{la}(\mathrm{z})$.

VERBENA HIRTA Spreng. 1966.

Additional bibliography: Moldenke, Phytologia 13: 249-250.

Reitz \& Klein 17484 represents the very conspicuously whitehirsute form or stage of this species; the flowers on their no. 17400 are described as "roxa".

Additional citations: BRAZIL: Paraná: Reitz \& Klein 17400 (W$2465578), 17484$ (Ac).

VERBENA HIRTA var. GRACILIS Dusén

Additional bibliography: Moldenke, Phytologia 13: 250. 1966.

The flowers on Reitz \& Klein 17883 are described as "roxa" it was collected at 950 meters altitude, blooming in Decenber. Additional citations: BRAZIL: Paraná: Reitz \& Klein 17616 (Ac), 17883 (Ac).

VERBENA HISPIDA RuIz \& Pav.

Additional bibliography: Bocq., Adansonia 2: 112 (1862) and 3: 203. 1863; Bocq., Rev. Verbenac. Ill2 \& 203. 1863; Moldenke, Phytologia 13: 250. 1966.

Recent collectors have found this plant growing among ancient Inca ruins along with Solamum raphanifolium, S. canasense, Cassia, Bidens, Mutisia, Baccharis, Oenothera, Calceolaria, Castilleja, Veronica, Euphorbia, Rumex, Capsella, Brassica, Plantago, Cerastium, Stellaria, Hypoxis, Lupinus, and species of non-tuberous Solanum, as well as in rocky soil along open roadsides with Solamum soukupii, Senecio, Tagetes, Capsella, Brassica, Lupinus, Cerastium, and non-tuberous species of Solanum, fruiting in February. Steinbach describes the flowers fflor violeta vivo base exterior púrpurea rojiso".

Additional citations: PERU: Cuzco: Ugent \& Ugent 3756 (Ws). Puno: Ugent \& Ugent 4543 (RP). BOLIVIA: Cochabamba: Ro F. Stein- 
bach $84(S)$.

VERBENA HOOKERIANA (Covas \& Schnack) Moldenke

Additional bibliography: Darlington \& Wylie, Chromosome Atl., pr. 1, 323 (1955) and pr. 2, 323. 1961; Moldenke, Phytologia 13: $250 \& 262$. 1966.

\section{XVERBENA HYBRTDA Voss}

Additional synonymy: Verbena hybrida Gronl. \& Rpl. ex Backer \& Bakh., F1. Java 2: 596. 1965 .

Additional bibliography: S. Sasaki, List P1. Formos. 353. 1928; Bodger, Electros Fls. 27, 61, 95, \& 105, pl. 54g, 4hle, 573c, 956a, $1137 f$, \& 3018f. 1952; Darlington \& Wyl1e, Chromosome Atl., pr. 1, 323 (1955) and pr. 2, 323. 1961; Clapham, Tutin, \& Warburg, F1. Brit. Isles, ed. 2, 729. 1962; Backer \& Bakh., F1. Java 2: 596. 1965; Noldenke, Phytologia 13: 250 \& 253. 1966; Mold enke, Résumés Supp1. 14: 10. 1966; Harborme in T. Swain, Compar. Phytochem. 285. 1966; Herbst Bros., Seeds for Nurserymen 23 \& 53. 1966.

Additional illustrations: Bodger, Electros Fls. pl. $54 \mathrm{hg}, 44 \mathrm{le}$, $573 \mathrm{c}$ [in color] 956a, 11371, \& $3018 \mathrm{~s}$ [in color]. 1952.

Sasald (1928) records the common names mizyo-zakura", "hanagasa", and "siki-zakura" for a plant which he calls V. phlogiflora Cham., cultivated in Formosa, but I suspect that his plant is actually xV. hybrida.

Bodger (1952) states that there are about 12,500 seeds of this plant per ounce. He lists V. hybrida grandiflora, V. grandiflora Mixed, V. grandiflora Brightness, and V. grandiflora Royale. He affirms that in southern California the average dates for planting this subject are January to February, the flowering takes place in July, and the harvesting of fruit in September, shipping of the seeds in October. The Herbst Brothers (1966) offer seeds of the following named varieties: Amethyst AAS 1966, Crystal (white), Dazzle, Delight (pink), Mixed, Red, Scarlet with purple eye, Splendor (purple and white), Starlight, and White. These are mostly described as dwarf forms, but also offered are Large-flowered Mixed and Early Dwarf Upright Rainbow Yixed.

\section{XVERBENA ILLICITA Moldenke}

Additional bibliography: Moldenke, Phytologia 13: 250. 1966.

Additional citations: IIIINOIS: Cálhoun Co.: A. R. Yoldenke 1275

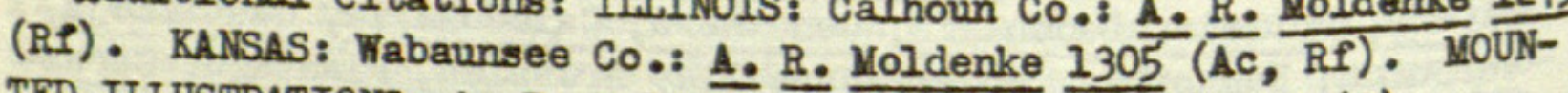
TED ILLUSTRATIONS: A. R. Moldenke Kod. I (z) $, \frac{1}{2(\mathrm{z})}, i_{3}(\mathrm{z})$.

XVERBANA INTERCEDENS Briq.

Additional bibliography: Moldenke, Phytologia 13: 250. 1966.

Woolston describes this plant as an herb, 1-1.5 m. tall, erect or falling over, the flowers pale-mauve, growing in wet places in low campos, and blooming in January.

Additional citations: PARAGUAY: Woolston 785 (S). 
VERBENA INTERUTEDIA Gill. \& Hook.

Additional bibliography: Darlington \& Wylie, Chromosome Atl., pr. 1, 323 (1955) and pr. 2, 323. 1961; Moldenke, Phytologia 13: 250. 1966.

\section{VERBBENA ILACINIATA (L.) Briq.}

Additional bibliography: Bocq., Adansonia 3: [Rev. Verbenac.] 203. 1863; Lefroy, Bull. U. S. Nat. Mus. 25: 96. 1884; Darlíngton \& Wylie, Chromosome Atl., pr. 1, 323 (1955) and pr. 2, 323. 1961; Backer \& Bakh., Fl. Java 2: 596. 1965; Solbrig, Castanea 30: 173174. 1966; Moldenke, Phytologia 13: 250-251, 262, \& 263. 1966.

The Verbena laciniata described by Backer \& Bakhuizen van den Brink (1965) from Javan gardens is V. tenuisecta Briq. as far as material seen by me is concerned. I doubt if the true $V_{0}$ laciniata occurs in Java. Bocquillon (1863) refers to V. laciniata as "V. erinoides Hook.", a binomial which I refer to $\bar{v} . \overline{\text { berterii }}$ (Yeisn.) Schau. Lefroy (1884) records V. laciniata from Bermuda, probably in error.

The A. Ruiz Leal $7665 / 14$, distributed as $\mathrm{V}$. laciniata, is actually $\overline{\text { V. }}$ glutinosa Kuntze.

VERBENA IAASIOSTACHYS Var. SCABRIDA Moldenke

Additional bibliography: Noldenke, Phytologia 11: 469. 1965; Hocking, Excerpt. Bot. A9: 364. 1965.

VERBENA IASIOSTACHYS var. SEPTENTRIONALIS Holdenke

Additional bibliography: Moldenke, Phytologia 11: 469. 1965; Hocking, Excerpt. Bot. A9: 364. 1965.

\section{VERBENA IIILACINA Greene}

Additional bibliography: Hocking, Excerpt. Bot. A9: 364. 1965; Moldenke, Phytologia 13: 20L-205. 1966.

\section{VERBENA LINDWAANII Briq.}

Additional bibliography: Hocking, Excerpt. Bot. A9: 364. 1965; Moldenke, Phytologia 13: 205. 1966.

VERBENA LIPOZYGIOIDES Talp.

Additional synonym: Verbena lipozygoIdes Walp. ex Bocq., Adan8onia 3: 203. 1863.

Additional bibliography: Bocq., Adansonia 3: [Rev. Verbenac.] 203. 1863; Moldenke, Phytologia 13: 251. 1966; Moldenke, Résumé Suppl. 14: 10. 1966.

VERBEHA LITORALIS H.B.K.

Additional bibliography: Bocq., Adansonia 3: [Rev. Verbenac.] 203. 1863; A. Stewart, Proc. Calif. Acad. Sci., ser. 4, 1: 134. 1911; Sampaio, Bol. Mus. Nac. Rio Jan. 13: 24, \& 21,1. 1937; Darlington \& wylie, Chromosome Atl., pr. 1, 323 (1955) and pr. 2, 323. 1961; Moldenke, Phytologia 13: 251-252. 1965; Noldenke, Résumé Supp1. 14: 2. 1966; J. G6mez, Biota 6: 107, 116, \& 119.'1966. 
Recent collectors have encountered this plant in pastures, along roadsides, in cloud-forests, and in somewhat dry roadsides with Caesalpinia, Salvia, Calceolaria, and Tagetes. Feddema refers to it as "an occasional weed".

Wiggins reports the chromosome count as $\mathrm{n}=21$ for his no. 18558 , but makes the qualifying statement "one cell 90 percent sure". On the Galapagos Islands the species has been found growing at the margins of trails in partial shade in the Scalesia zone, in partial shade among trees and shrubs, and "scattered in the Scalesia Zone". The flowers are described as "lavender" on I. L. Wiggins 18508, on Breedlove 11634 they were mblue", on Dressler \& Jones 177 "blue-violet", and on Feddema 1667 "corolla pale-lavender, throat darker purple-lavender".

Sampaio (1937) regards V. brasiliensis Veli. as synonymous with V. litoralis and records the vernacular names "herva do pai Caetano" and "herva de pai Caetano".

A. R. Koldenke 1232, cited below under V. parvula Hayek, is regarded by my son (the collector) as a mere ecotype of V. litoralis. He tells me that $V$. litoralis was growing commonly all through the area where this collection was made, with varying forms of stature, and that the above-mentioned collection merely represents its smaliest alpine form. If this is an accurate description of the situation, then it is possible that Hayek's binomial should be reduced to form status under V. litoralis.

Additional citations: MEXrCO: Michoacán: Feddema 1667 (Mi). Vera Cruz: Dressler \& Jones 177 (Mi). GUATFiHAIA: Huehuetenango:

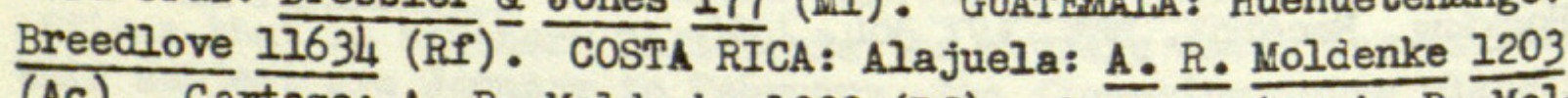
(Ac). Cartago: A. R. Moldenke 1320 (Rf). Guanacaste: A. R. Moldenke 1220 (Ac). Limbn: A. R. Moldenke 1319 (Rf). San José: A. R. Moldenke 1201 (Ac). COIOMBIA: Cundinamarca: Larsson s.n. "Quetame, 0ct. 1964] (S). PERU: Cajamarca: Ugent \& Ugent 5512 (AC). GALAPAGOS ISLANDS: Charles: Itow 179 (Du-564532). Indefatigable: R. I. Bowman s.n. [0ct. 5/57] (Gg-461948); I. L. Wiggins 18508 (Du $=564528), \underline{18558}(\mathrm{Du}-564539)$.

\section{VERBENA LONGIFOLIA Yart. \& Gal.}

Additional bibliography: Moldenke, Phytologia 13: 251 \& 252. 1966; Loldenke, Résumé Suppl. I14: 2. 1966.

VERBENA LONGIFOLIA var. PUBESCENS Moldenke
Bibliography: Moldenke, Phytologia 13: 307. 1966; Moldenke, Resume Supp1. 14: 2. 1966.

Citations: MEXICO: Oaxaca: Breedlove 12292 (Z-type).

VERBENA LUCANENSIS Moldenke

Additional bibliography: Hocking, Excerpt. Bot. A9: 365.1965 ;

Moldenke, Phytologia 13: 207. 1966. 
VERBENA MACDOUGAIII Heller

Additional bibliography: Hocking, Excerpt. Bot. A9: 365. 1965; Moldenke, Phytologia 13: 208. 1966.

VERBENA MACROSPERMA SpEg.

Additional bibliography: Moldenke, Phytologia 10: 119-121. 1964; Hocking, Excerpt. Bot. A9: 365.1965.

VERBENA MARITIMA SmaIl

Additional bibliography: Hocking, Excerpt. Bot. A9: 365. 1965; Moldenke, Phytologia 13: 208. 1966.

VERBENA MARRUBIOIDES Cham.

Additional bibliography: Hocking, Excerpt. Bot. A9: 365. 1965; Moldenke, Phytologia 13: 208. 1966.

XVERBENA MATRITENSIS Moldenke

Additional bibliography: Moldenke, Phytologia 10: 129. 1964; Hocking, Excerpt. Bot. A9: 365. $1965^{\circ}$.

VERBENA MEGAPOTAMICA Spreng.

Additional bibliograohy: Darlington \& Wylie, Chromosome Atl., pr. 1, 323 (1955) and pr.2, 323. 1961; Hocking, Excerpt. Bot. A9: 365. 1965; Moldenke, Phytologia 13: 208-209. 1966.

VERBENA MENDOCINA R. A. Phil.

Additional bibliography: Darlington \& Wylie, Chromosome Atl., pr. 1, 323 (1955) and pr. 2, 323. 1961; Moldenke, Phytologia 13: 252 \& 271.1966.

VERBENA MENTHAEFOLIA Benth.

Additional bibliography: Moldenke, Phytologia 13: 252 \& 276. 1966; Holdenke, Résumé Supp1. 14: 2. 1966.

Recent collectors have encountered this plant in grazed areas near roadsides, on steep slopes with Quercus and Pinus, in cornfields, and in weedy patches between irrigation ditches, growing in association with Solanum michoacanum, S. bulbocastanum, S. stoloniferum, Physalis, Gaura, Oenothera, and Hypericum.

Additional citations: IEXICO: Chiapas: Breedlove 11161 (Ac). Michoacán: Ugent, Ugent, \& Flores C. 5802 (Ws). Sinaloa: Feddema 1808 (Mi).

VERBENA MICROPHYLITA H.B.K.

Additional bibliography: Bocq., Adansonia 3: [Rev. Verbenac.] 203. 1863; Moldenke, Phytologia 13: 252, 253, 262, \& 271.1966.

Recent collectors have found this plant growing along lake shores, in open village fields, on bare clay banks with Solanum acaule, and on vast Ichu-tussock grasslands with a few small round cacti, Solanum acaule, Tetraglochin (strictum?), and a fer other small shrubs, with calcareous outcrops. 
Additional citations: PERU: Puno: Iltis \& Iltis s.n. [10 km. SW. of Puno on road to Llave, Jan. 10, 1963] (Ac); Iltis, IItis, Ugent, \& Ugent 1376 (Ws); Iltis \& Ugent 1393 (RF).

VERBENA MINUTIFLORA Briq.

Additional bibliography: Moldenke, Phytologia 13: 252. 1966;

G. Taylor, Ind. Kew. Suppl. 13: 143.1966.

The flowers on Reitz \& Klein 17805 are described as "roxa". Additional citations: BRAZIL: Paraná: Reitz \& Klein 17805 (Ac).

XVERBENA MOECHINA Moldenke

Additional bibliography: E. W. Lathrop, Univ. Kans. Sci. Bull. 39: 173. 1958; Hocking, Excerpt. Bot. A9: 365. 1965; Moldenke, Phytologia 13: 210. 1966.

Lat hrop (1958) states that this plant may be looked for in prairle pastures and on open wooded hillsides in Kansas.

VERBEANA MONTEVIDENSIS Spreng.

Additional bibliography: Darlington \& Wylie, Chromosome Atl., pr. 1, 323 (1955) and pr. 2, 323. 1961; Moldenke, Phytologia 13: 252. 1966.

VERBENA NEOMEXICANA (A. Gray) Small

Additional bibliography: Hocking, Excerpt. Bot. A9: 365. 1965; Moldenke, Phytologia 13: 2il. 1966.

VERBEIIA NEOUKEXICANA var. HIRTELIIA Perry

Additional bibliography: Hocking, Excerpt. Bot. A9: 365. 1965; Moldenke, Phytologia 13: 211. 1966.

The flowers are described as "lavender" on D. S. Correll 33855. The plant has been found growing on talus slopes. The G. L. Webster $\frac{453}{\text { Greene. }}$ distributed as this variety, is actually V. plicata Additional citations: TEXAS: Brewster Co.: D. S. Correll
$\underline{33855}$ (Ld).

VERBENA NEOMEXICANA Var. IYLOPODA PErry

Additional bibliography: Hocking, Excerpt. Bot. A9: 365.1965 ; Moldenke, Phytologia 13: 211. 1966.

VERBENA OFFICINAIIS I.

Additional \& emended bibliography: ROhl., Deutschl. Fl., ed. 1, 2: 329.1812 ; W. D. J. Koch in Rohl., Deutschl. FI., ed. 2, 4: 339. 1833; W. D. J. Koch, Syn. FI. Germ., ed. i, 578. 1837; W. D. J. Koch, Syn. Deutsch. \& Schw. FI., ed. 1, 575-576. 1838; Neigen, Deutschi. FI. 3: 130. 1842; W. D. J. Koch, Taschenb. Deutsch. FI., ed. 1, 417. 1843; W. D. J. Koch, Syn. Fl. Germ., ed. 2, 2: 664. 1844; W. D. J. Koch, Syn. Deutsch. \& Schw. FI., ed. 2, 681-682. 1846; G. \& F. Lorinser, Taschenb. F1. Deutschl., ed. 1, 311 (1847) and ed. 2 , 3il. 1851; W. D. J. Koch, Taschenb. Deutsch. FI., ed. 3, 417 (1851) and ed. 4, 400. 1856; Langmann, F1. Nord- \& Vittel- 
deutsch., ed. 2, 252-253. 1856; W. D. J. Koch, Taschenb. Deutsch. Fl., ed. 5, 400. 1860; Bocq., Adansonia 2: $107 \& 154$ (1862) and 3: 203. 1863; Bocq., Rev. Verbenac. 107, 154, \& 203. 1863; W. D. J. Koch, Taschenb. Deutsch. FI., ed. 6, 400. 1865; Langethal, Beschreib. Gew. Deutsch., ed. 2, 448 . 1868; Miq., Cat. Mus. Bot. Iugd Bat. 70. 1870; E. Hall. in W. D. J. Koch, Taschenb. Deutsch FI., ed. 7, 403 (1878) and ed. 8, 403. 1881; K. G. Lutz, Pflanzenfr. 93, pl. 17, fig. 19. 1887; W. Medic., Illustr. Pflanzenb. vili, codx, \& 115, pl. 50, fig. 112 \& 112a. 1893; J. Matsum., Bot. Mag. Tokgo 13: [113]-114. 1899; Ichimura, Bot. Mag. Tokyo 13: 116. 1899; Miguia, Exkursionsfl. Deutsch.' 2: 134. 1906; Kawakami, List P1. Formos . 85. 1910; A. Stewart, Proc. Calif. Acad. Sci., ser. 4, 1: 134. 1911; Dunn \& Tutcher, Kew Bull. Misc. Inf. Addit. Ser. 10: 202. 1912; Turrill, Kew Bull. Misc. Inf. 1924: 359. 1924; S. Sasaki, List PI. Formos. 353. 1928; Hand.-Mazz., Ann. Hort. Gothenb. 9: [67]. 1934; Fletcher, Kew Buil. Misc. Inf. 1938: 410-411. 1938; Blakelock, Kew Bull. Misc. Inf. 1949: 539. 1949; K. H. Rechinger, Arkiv Bot., ser. 2 2: 408. 1952; Bor \& Raizada, Some Beaut. Ind. Climbers [136]. 1954; Darlington \& Wylie, Chronosome Atl., pr. 1, 323. 1955; De Mire \& Gillet, Journ. Agric. Trop. 3: 721. 1956; Mostert, Bot. Sury. S. Afr. Mem. 31: 41 \& 191. 1958; T. A. Rao, Bull. Bot. Surv. Ind. 1: 114. 1959; K. H. Rechinger, Arld $\nabla$ Bot., ser. 2, 5: 346. 1959; Anon., Kem Bull. Gen. Index 1929-1956, 291. 1959; Nath, Bot. Surv. South. Shan States 143. 1960; Darlington \& Wille, Chromosame Atl., pr. 2, 323. 1961; Clapham, Tutin, \& Warburg, Fl. Brit. Isles, ed. 2, 730. 1962; Lid, Norsk \& Svensk. F1. 568 \& 569 , fig. 34la. 1963; Quezel \& Santa, Nouv. Fl. Algérie 2: 780. 1963; Hepper in Hutchinson \& Dalz., Fl. T. Trop. Afr., ed. 2, 2: 434. 1963; Backer \& Bakh., F1. Java 2: 596. 1965; Moldenke, Phytologia 13: 253. 1966; Kitamura, Results Kyoto Univ. Scient. Exped. Karakoram 8: 132. 1966; Rickett, Wild Fls. U. S. I (2): 362. 1966; G. B. Foster, Herbs for Every Gard. 199. 1966; Ross-Craig, Drawings Brit. Pl. 23: pl. 38. 1966.

Additional illustrations: K. G. Lutz, Pflanzenfr. pl. 17, flg. 19 [in color]. 1887; W. Medic., Illustr. Pflanzenb. pl. 50, fig. 112 \& $112 \mathrm{a}$ [in color]. 1893; Lid, Norsk \& Svensk. FI. fig. 34 la. 1963; Ross-Craig, Drawings Brit. P1. 23: pl. 38 • 1966.

Rao (1959) describes this plant as an "herb with many long spikes bearing white flowers", growing at 1300 to 1615 meters altitude in India, and cites his no. 6539. Mostert (1958) avers that it grows on streambanks, dams, pans, flooded and irrigated areas subject to wetness and drought, severe grazing, and trampling, and is "comnon in wet situations" in South Africa. RossCraig (1966) describes the corolla as "very delicate lilac-mauve", with the "lower part of tube pinkish-crimson" and the leaves "bright medium-green", paler beneath. Lid (1963) records the Scandinavian names "jernurt" and "jetrnort". Formosan common names are "bé-pin-chhau", "kumatudura", "kuma-tzurzura", "marupoyopoyomu", "t8-bi-chhán", "thih-be-pin", and "thih-tiò-koan".

Nath (1960) records the species as both cultivated and escaped in Burma; Quezel \& Santa (1963) say that it is found throughout 
Algeria, that it is a paleo-temperate plant, and that it is called "ben noul" there. Blakelock (1949) cites Guest \& Iudlow-Hewitt 2910, 2994, \& 3041 and Lazar 3442 \& 3638 from Iraq.

The Felizardo s.n., distributed as $V_{0}$ officinalis, is actually something in the Rubiaceae.

Additional citations: JUGOSLAVIA: Istria: Starbatck J.17 (S). ZAMBIA: E. A. Robinson 5596 (S).

VERBENA OVATA Cham.

Additional bibliography: Darlington \& Wrlie, Chromosome Atl., pr. 1, 323 (1955) and pr. 2, 323. 1961; Moldenke, Phytologia 13: 213. 1966.

Woolston describes this plant as an erect herb, with mauve flowers.

Additional citations: PARAGUAY: Woolston 916 (S).

VERBENA PARANENSIS Moldenke

Additional bibliography: Moldenke, Phytologia 13: 213. 1966;

G. Taylor, Ind. Kew. Supp1. 13: 143.'1966.

VERBENA PARODII (Covas \& Schnack) Koldenke

Additional bibliography: Darlington \& Wylie, Chromosome Atl., pr. 1, 323 (1955) and pr. 2, 323. 1961; Noldenke, Phytologia 13: 252, 253, \& 271. 1966; Moldenke, Résumé Suppl. I 14.1966.

\section{VERBENA PARVULA Hayek}

Additional bibliography: Moldenke, Phytologia 10: 297 (1964) and 13: 214. 1966.

Recent collectors have encountered this species on high alpine valley floors, on sunny lichen-covered rock-walls, rock-piles, terraces, and cliffs among ancient Inca ruins, growing in association with Solanum raphanifolium, S. canasense, Cassia, Bidens, Mutisia, Baccharis, Oenothera, Calcoolaria, Castilleja, Veronica, Euphorbia, Rumex, Capsella, Brassica, Plantago, Cerastium, SteIlaria, Hypoxis, Lupinus, and non-tuberous species of Solanum.

Iltis \& Ugent describe the plant as having weak and more or less decumbent branches, and report it as a common weed in pastures and fallow vegetable garden fields, flowering in December, fruiting in May and December. The flowers on Saunders $\frac{363}{60}$ are said to have been purple, while those on Iltis \& Ugent
"violet".

The Hammarlund 167 \& 196, cited by me in Phytologia 10: 297 (1964), prove on re-examination to be $V$. weberbaueri Hayek.

Iy son, Andrew R. Moldenke, who collected a specimen of what appears to me to be $\mathrm{V}$. parvula for the first time in Costa Rica, is of the opinion that his specimen, at least, represents merely an alpine ecotype of V. litoralis H.B.K. He affirns that the latter species was common in the area and of various forms of stature, and that his collection represents merely the most dwarf form seen. If this is correct, then perhaps Hayek's species ought 
to be reduced to form rank under V. litoralis; or perhaps the Costa Rican plant is not identical with the South American plant passing as $\mathrm{V}$. parvila.

Additional citations: COSTA RICA: Cartago: A. R. Moldenke 1232 (Rf). PERU: Apurimac: Iltis \& Ugent 699 (Rf). Cuzco: D. Ugent $\frac{5350}{\text { (Mi). (Ws); Ugent \& Ugent } 3749}$ (AC). Lima: S. G. E. Saunders 363

\section{VERBENA PERAKII (Covas \& Schnack) koldenke}

Additional bibliography: Darlington \& Wylie, Chromosome Atl., pr. 1, 323 (1955) and pr. 2, 323. 1961; Moldenke, Phytologia 13: 214. 1966.

\section{VERBENA PERUVIANA (L.) Britton}

Additional bibliography: Bocq., Adansonia 2: 107 (1862) and 3: 202. 1863; Bocq., Rev. Verbenac. 107 \& 202. 1863; Gamble, F1. Madras 1106. 1924; Sampaio, Bol. Mus. Nac. Rio Jan. 13: 190. 1937; Darlington \& Wylie, Chromosome Atl., pr. I, 323 (1955) and pr. 2, 323. 1961; Clapham, Tutin, \& Warburg, FI. Brit. Isles, ed. 2, 729. 1962; Backer \& Bakh., Fl. Java 2: 596. 1965; Hocking, Excerpt. Bot. A9: 365. 1965; Moldenke, Phytologia 13: 215. 1966.

Sampaio (1937) records for this plant the additional vernacular name "jurujuba".

\section{VERBENA PERUVIANA $f$. ALBA Moldenke}

Additional bibliography: Hocking, Excerpt. Bot. A9: 365. 1965; Koldenke, Phytologia 13: 215. 1966.

VERBENA PERUVIANA var. GLABRIUSCULA Kuntze

Additional bibliography: Hocking, Excerpt. Bot. A9: 365 \& 366 . 1965; Moldenke, Phytologia 13: 215-216. 1966.

\section{VERBENA PHLOGIFLORA Cham.}

Additional bibliography: Bocq., Adansonia 3: [Rev. Verbenac.] 203. 1863; S. Sasaki, List P1. Formos. 353. 1928; Darlington \& Wylie, Chromosome AtI., pr. 1, 323. 1955; Nath, Bot. Surv. South. Shan States 143. 1960; Darlington \& Wylie, Chromosome Atl., pr. 2, 323. 1961; Moldenke, Phytologia 13: 253. 1966.

The flowers on Reitz \& Klein 17786 are described as "roxa". Sasaki (1928) reports the Japanese common names "bizyo-zakura", "hana-gasa", and "siki-zakura", but I suspect that the plants to which these names are applied are actually xV. hybrida Voss. Similarly, Nath (1960) records $V$. phlogiflora as cultivated in Burna, but, here again, he is probably referring to the commonly cultivated $x V$. hybrida.

Additional citations: BRAZIL: Paraná: Reitz \& Klein 17786 (Ac).

VGRBENA PLATENSIS Spreng.

Additional bibliography: Bocq., Adansonia 3: [Rev. Verbenac.] 203. 1863; Darlington \& Wylie, Chromosome Atl., pr. 1, 323 (1955) 
and pr. 2, 323. 1961; Moldenke, Phytologia 13: 254.1966.

VERBENA PLICATA Greene

Additional bibliography: Moldenke, Phytologia 13: 247, 248, \& 254. 1966; Moldenke, Résumé Suppl. I4: 1 \& 10. 1966.

Webster found this species growing in mesquite scrab.

The B. E. Holland 2I, distributed as V. plicata, is actually

V. canescens var. roemeriana (Scheele) Perry.

Additional citations: TEXAS: Brewster Co.: D. S. Correll 34072 (Id). Brown Co.: R. Fryar 28 (Id). Terreli Co.: G. L. Webster 453 (Mi).

VERBENA POGOSTOMA Klotzsch

Additional bibliography: Moldenke, Phytologia 13: 217-218 \& 271. 1966.

VERBENA PULCHETILA Sweet

Additional bibliography: Bocq., Adansonia 2: 106, 107, 131, \& 132. 1862; Bocq., Rev. Verbenac. 106, 107, 131, \& 132. 1863; Moldenke, Phytologia 13: 254-256, 261-263, \& 267-272. 1966; yoldenke, Résumé Suppl. 14:9. 1966; Solbrig, Castanea 30: 173-174. 1966.

VERBENA PULCHELIA var. CLAVFLIATA (Troncoso) Shinners

Synonymy: Glandularia pulchella var. clavellata Troncoso, Darwiniana 13: $4 \overline{75 \text { \& } 476 \text {, fig. } 3.1964 .}$

Bibliography: Troncoso, Darwiniana 13: 475 \& 476 , fig. 3. 1964; Shinners, Sida 2: 266. 1966; Moldenke, Phytologia 13: 255. 1966; Noldenke, Résumé Supp1. 14:9. 1966.

Illustrations: Troncoso, Darwiniana 13: 475, fig. 3. 1964 .

VERBENA PUMILA RYdb. 1966.

Additional bibliography: Moldenke, Phytologia 13: 256-257.

Additional citations: TExAS: Bromn Co.: Faubion 6 (Id). Foard Co.: C. Turner 팬 (Id). Henderson Co.: C. Turner 5 (Id). San Saba Co.: CaIhoun 9 (Id).

VERBENA PUMTILA f. AIBIDA Moldenke 1966.

Additional bibliography: Holdenke, Phytologia 13: 256 \& 257 .

VERBENA QUADRANGULATA Heller

Additional bibliography: Moldenke, Phytologia 13: 247 \& 257258. 1966.

VERBENA RACENOSA Eggert

Additional bibliography: Darlington \& Wylie, Chromosome Atl., pr. 1, 323 (1955) and pr. 2, 323. 1961; Yoldenke, Phytologia 13:
258. 1966. 
VERBENA RADICATA Moldenke

Additional bibliography: Darlington \& Wylie, Chromosome Atl., pr. 1, 323 (1955) and pr. 2, 323. 1961; Loldenke, Phytologia 13: 258. 1966.

VERBEANA RAMBOI Moldenke 1966.

Additional bibliography: Moldenke, Phytologia 13: 258 \& 264 .

VERBENA RECTA H.B.K.

Additional bibliography: Moldenke, Phytologia 13: 258. 1966.

Rzedowski encountered this plant in open pinewoods with abundant grasses, at 3000 meters altitude.

Additional citations: MEXICO: Federal District: J. Rzedowski $20443 \mathrm{a}$ (Mi).

VERBENA REITZII Moldenke

Additional bibliography: Moldenke, Phytologia 13: 258-259. 1966; Moldenke, Résume Suppl. 14: 3.1966.

VERBENAA REITZII var. CASTRENSIS Moldenke

Additional bibliography: Moldenke, Phytologia 13: 259. 1966; Yoldenke, Résumé Suppl. 14: 3. 1966.

VERBENA RIBIFOIIA Walp.

Additional bibliography: Bocq., Adansonia 3: [Rev. Verbenac.] 203. 1863; Moldenke, Phytologia 13: 259. 1966.

VERBENA RIGIDA Spreng.

Additional synonymy: Verbena venosa Hook. ex Bocq., Adansonia

3: 203. 1863.

Additional bibliography: Bocq., Adansonia 3: [Rev. Verbenac.] 203. 1863; Gamble, FI. Madras 1706. 1924; Darlington \& Wylie, Chromosome Atl., pr. i, 323 (1955) and pr. 2, 323. 1961; Backer \& Bakh., FI. Java 2: 596. 1965; Moldenke, Phytologia 13: 259-260. 1966; Uoldenke, Résumé SuppI. 14: I \& 10. 1966.

In Jamaica recent collectors have found this plant growing at 2600 to 3400 feet altitude, and describe it as "common on roadsides". The flowers on Webster \& Proctor 5249 are described as "lavender", while Crosby, Hespenheide, \& Anderson 427 had "calyx green below to tinged purple above, corolla purple, white where covered by calyx, the hairs white, fllaments white, anthers light yellow-green, style white below to purplish near stigma, stigma purplish".

Additional citations: GEORGIA: Baldwin Co.: H. N. Moldenke 23036 (Ac). JAMAICA: Crosby, Hespenheide, \& Anderson 427 (Vi); Webster \& Proctor 5249 (Mi). BRAZII: Paranăa: Reitz \& Klein $\underline{17672(\mathrm{Ac})}$.

VERBENA RIPARIA Raf.

Additional bibliography: Moldenke, Phytologia 11: 479. 1965; 
Rickett, Wild Fls. U. S. I (2): 362. 1966.

VERBENTA RUNYONI Moldenke

Additional bibliography: Koldenke, Phytologia 13: 260. 1966.

Runyon describes this plant as a "tall erect plant with opposite leaves and hollow stems", scarce on clay soil in damp situations. Additional citations: TEXAS: Cameron Co.: R. Runyon 6017 (Ld).

XVERBENA RYDBERGII Moldenke

Additional bibliography: E. W. Lathrop, Univ. Kans. Sci. Bull. 39: 173. 1958; Moldenke, Phytologia 13: 260-261. 1966.

One of the A. R. Moldenke 819 specimens, cited previously as deposited in my personal herbarium, is now in that of the Texas Research Foundation at Renner, Texas. Lathrop (1958) tells us that this plant may be expected in overgrazed prairie pastures, prairie woodlands, and waste places in Kansas, and this agrees completely with the observations of my son and myself.

Additional citations: KANSAS: Dickinson Co.: A. R. Moldenke 1298 (Rf). Jackson Co.: A. R. Moldenke 1314 (AC). Osage Co.: A. R. Moldenke 1304 (AC). MISSSOURI: Monroe Co.: A. R. Moldenke $12 \overline{80}$

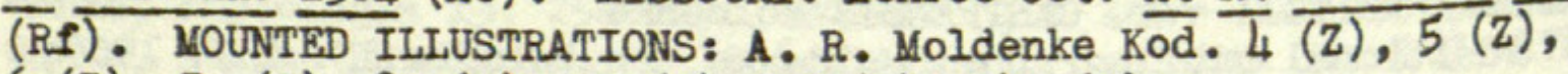
$6(\mathrm{z}), 7 \mathrm{a}(\mathrm{z}), 8 \mathrm{a}(\mathrm{z}), 10(\mathrm{z}), 12(\mathrm{z}), 14 \mathrm{~b}(\mathrm{z})$.

\section{VERBENA SANTIAGUENSIS (Covas \& Schnack) Moldenke}

Additional bibliography: Darlington \& Wylie, Chromosome Atl., pr. 1, 323 (1955) and pr. 2, 323. 1961; Moldenke, Phytologia 13: 261. 1966.

VERBENA SCABRA Vahl

Additional bibliography: Moldenke, Phytologia 13: 261. 1966; Rickett, Wild Fls. U. S. I (2): 364. 1966.

In Jamaica this species is reported to be "comnon in the drier areas of swampy country". The flowers on Webster \& Proctor 5334 are described as "lavender", while those on Webster \& Wilson 5051 plant as an annual.

Additional citations: LOUISIANA: Orleans Par.: T. Drummond s.n. [New Orleans, 1833] (s). JAMAICA: Webster \& Proctor 5334 (Mi); Webster \& Wilson 5051 (Mi).

VERBENA SCABRA $f$. ANGUSTIFOLIA Moldenke, $f$. nov.

Haec forma a forma typica spociei laminis foliorum anguste vel angistissime ellipticis vel elliptico-lanceolatis recedit.

This form differs from the typical form of the species in having its leaf-blades narrowly or very narrowly elliptic or elliptio lanceolate, $3-6.5 \mathrm{~cm}$. long, $0,5--2 \mathrm{~cm}$. wide, attenuate-acute at the apex, with the margins sharply and irregularly mucronulateserrate.

The type of the form was collected by John R. Crutchfield (no. 1837) in moist black loam at the edge of Beaver Creek, Burnet 
County, Texas, on July 21, 1966, and is deposited in the Lundell Herbarium at Renner, Texas. The collector states that the flowers are light-pink. While leaves approximating these in size and shape are sometimes seen in and near the young inflorescences in the typical form of the species, in the present form they are uniform to the base of the plant. type).

Citations: TEXAS: Burnet Co.: J. R. Crutchfield 1837 (Ld-

VERBENA SEDULA Moldenke 1964 .

Additional bibliography: Koldenke, Phytologia 11: 140-114.

Bowman found this plant growing at 2500 feet altitude on the north slope of the main peak on Indefatigable Island, with white flowers in February. Material has been misidentified and distributed in herbaria as $\mathrm{V}$. carolina $\mathrm{L}$.

Additional citations: GALAPAGOS ISLANDS: Indefatigable: R. I. Bownan 81 (Gg-461129).

VERBENA SELLOI Spreng.

Additional bibliography: Moldenke, Phytologia 13: 247, 248, $251,255,256,261-264,267,270, \& 271$. 1966; Moldenke, Résumé Suppl. 14: $3,5, \& 9.1966$.

\section{VERBENA SIMPLEX Lehm.}

Additional bibliography: Bocq., Adansonia 2: 113 (1862) and 3: 202. 1863; Bocq., Rev. Verbenac. 173 \& 202. 1863; W. Stone, Ann. Rep. N. J. State Mus. 1910: 661. 1911; Brehan, Kew Bull. Nisc. Inf. 1950: 226. 1950; E. W. Lathrop, Univ. Kans. Sci. Bull. 39: 173. 1958; Moldenke, Phytologia 13: 251, 264, \& 265. 1966; Rickett, Wild Fls. U. S. I (2): 362, pl. 112 . 1966.

Additional illustrations: Rickett, Wild Fls. U. S. I (2): pl. 112 [in color] 1966.

Lathrop (1958) tells us that in Kansas this plant may be found in prairie pastures, rocky prairie woodlands, and open oak woods.

\section{XVERBENA SOLBRIGII Moldenke}

Additional bibliography: Moldenke, Phytologia 13: 264. 1966; G. Taylor, Ind. Kew. Suppl. 13: 143.1966.

VERBENA STELLARIOIDES Cham.

Additional bibliography: Moldenke, Phytologia 13: 258 \& 264265. 1966.

\section{VERBENA STRICTA Vent.}

Additional bibliography: Bocq., Adansonia 3: [Rev. Verbenac.] 203. 1863; C. Robertson, Psyche 29: 159-173. 1922; E. W. Lathrop, Univ. Kans. Sei. Bull. 39: $12 \mathrm{~L}_{4} \& 173.1958$; Ungar, Univ. Kans. Sci. Bull . 46: 28. 1965; Percival, Flor. Biol. 131. 1965; R. B. Clarkson, Castanea 31: 100. 1966; Rickett, Wild Fls. U. S. 1 (2): 362, p1. 112. 1966; Moldenke, Phytologia 13: 260, 261, \& 265.
1966. 
Additional illustrations: Rickett, Wild Fls. U. S. I (2): pl. 112 [in color] 1966.

Robertson (1922) and Percival (1965) report that the oligolectic bee, Verbenajus verbenae, a member of the family Panurgidae, collects only from this species, V. hastata L., and V. urticifolia L.

Lathrop (1958) states that in Kansas $V$. stricta is found in rocky prairie gullies, overgrazed pastures, fields, and open floodplain woods, while Ungar (1965) found it in the hemicryptophyte formation on prairies and freshwater seeps.

Additional citations: ILIINOIS: Calhoun Co.: A. R. Moldenke 1274 (AC). Greene Co.: A. R. Moldenke 127 (Rf) - Macoupin Co.: A. R. Moldenke 1266 (Ac) - Montgomery Co.: A. R. Moldenke 1264 (Rf). KANSAS: Dickinson Co.: A. R. Moldenke 1299 (Ac, Rf). Nemaha Co.: H. N. Moldenke $23128(\overline{A c}, \overline{R f})$. Shawnee Co.: H. N. Moldenke 23140 (Rf). Wabaunsee Co.: A. R. Moldenke 1288 (Rf). NEBRAS$\overline{\mathrm{KA}}:$ Buffalo Co.: Guthe s.n. [Kearney, July 1893] (Mi). MOUNTED ILLUSTRATIONS: A. R. Moldenke Kod. $3(\mathrm{z}), \eta_{\mathrm{a}}(\mathrm{z}), \mathrm{I}_{4} \mathrm{a}(\mathrm{z}), 15$ (2).

VERBENA STRIGOSA Cham.

Additional bibliography: Bocq., Adansonia 3: [Rev. Verbenac.] 203. 1863; Moldenke, Phytologia 13: 265-266. 1966.

VERBENA SUBINCANA (Troncoso) Shinners

Additional bibliography: Loldenke, Phytologia 13: 255, 256, 266-268, \& 270. 1966; Yoldenke, Résumé Suppl. 14:9. 1966.

VERBENA SULPHUREA D. Don

Additional bibliography: Bocq., Adansonia 2: 126 (1862) and 3: 203. 1863; Bocq., Rev. Verbenac. I26 \& 203. 1863; Moldenke, Phytologia 13: 268 . 1966.

VERBENA SUPINA I.

Additional bibliography: Meigen, Deutschl. F1. 3: 130. 1842; Bocq., Adansonia 3: [Rev. Verbenac.] 203. 1863; Blakelock, Kew Bull. Misc. Inf. 1949: 539. 1949; Berhaut, F1. Sénegal 65. 1954; Anon., Kew Bull. Gen. Index 1929-1956, 291. 1959; Quezal \& Sants, Souv. F1. Algérie 2: 780. 1963; Hepper in Hutchinson \& Dalz., F1. W. Trop. Afr., ed. 2, 2: 434. 1963 ; Moldenke, Phytologia 13: 268. 1966; Anon., Assoc. Etud. Tax. F1. Afr. Trop. Index 1965: 67. 1966.

Quezel \& Santa (1963) state that this plant is found throughout Algeria, is Mediterranean in general distribution, and is called "djaida" in Algeria. Hepper (1963) describes the species as follows and cites the following specimens: "A small annual herb 2-12 in. tall, appearing gray-green; flowers very small, lilac; in seasonaliy flooded ground: MAURITANIA: Popov 15. SENEGAL: Berhaut 554. MALI: Davey 440 ; also in Sudan, Ethiopia, and around the Mediterranean." Blakelock (1949) cites Lazar 1181 from Iraq. 
VERBENA SUPINA f. ERECTA Moldenke

Additional bibliography: Moldenke, Phytologia 11: 481. 1965; Anon., Assoc. Etud. Tax. Fl. Afr. Trop. Index 1965: 67. 1966.

VERBENA TENERA Spreng.

Additional bibliography: Bocq., Adansonia 3: [Rev. Verbenac.] 203. 1863; Darlington \& Wylie, Chromosome Atl., pr. 1, 323. 1955; Mostert, Bot. Surv. S. Afr. Mem. 31: 41 \& 191. 1958; Darlington \& Wylie, Chromosome Atl., pr. 2, 323. 1961; Hepper in Hutchinson \& Dalz ., Fl. W. Trop. Afr., ed. 2, 2: 434. 1963; Moldenke, Phytologia 13: $214,217,252,253,255,256,262,263, \& 268-273.1966$.

liostert (1958) speaks of this species as a garden escape in South Africa, but he is probably referring to V. tenuisecta Briq, a far more widely cultivated species. Hepper $\overline{(1963) \text { refers to }}$ V. tenera as $n_{2}$ creeping herb with very dissected leaves and white or violet flowers" and notes that it "has been introduced into Nigeria (Obubra Dist., FHI 43964)n, but here again it is probably $\mathrm{V}$. temuisecta that is the species involved.

VERBEENA TENERA var. ALBIFLORA Kuntze 1965.

Additional bibliography: Moldenke, Phytologia 11: 253 \& 276.

The Venturi 5267, cited by me as this variety in Phytologia 11: 276 (1965), is actually V. parodii (Covas \& Schnack) Moldenke.

VERBEANA TENUISECTA Briq.

Additional bibliography: Mostert, Bot. Surv. S. Afr. Mem. 31: 41 \& 191. 1958; Hepper in Hutchinson \& Dalz., F1. W. Trop. Afr., ed. 2, 2: 434. 1963; Liogier, Rhodora 67: 349. 1965; Solbrig, Castanea 30: 173-174. 1966; Moldenke, Phytologia 13: 246, 255, 262, 263, \& 270-273. 1966; Moldenke, Résumé Suppl. 14: I \& 10 .

The flowers on Reitz \& Klein 17728 are described as "roxa". Mostert (1958) refers to V. tenera Spreng. as a garden escape in South Africa, but is probably referring to V. temuisecta. Hepper (1963) speaks of a $\nabla$. tenera Spreng. Introduced into Nigeria "a creeping herb with very dissected leaves and white or violet flowers", but the plant to which he refers is almost certainly temuisecta. He cites FHI 43964 from the Obubra District.

Woolston refers to $\frac{1}{V_{0}}$ tenuisecta as a "semi-procumbent herb." Additional citations: TEXAS: Harris Co.: H. Spoon 2l (Id). BRAZIL: Paraná: Reitz \& Klein 17728 (Ac). PARAGÜAY: Woolston 254 (S).

VERBENA TEUCRIIFOIIA Mart. \& Gal. 1966.

Additional bibliography: Moldenke, Phytologia 13: 273-274.

Recent collectors have found this plant growring at the edges of Pimus-Quercus woods and grazed fields, along rock-brush walls with Solanum stenophyllidium, S. Inscendens, and Rubus, and in oak 
Vol. 14, no. 5

woods with Cassia, Solanum stoloniferum, S. skutchii, S. appendiculatum, S. rostratum, and very many different kinds of herbaceous and tree-like species of Solamum.

Additional citations: $\overline{M E X I C O}$ : Michoacán: Feddema 1684 (Mi); Ugent \& Flores C. 2165 (Ws). Vera Cruz: Ugent \& Flores C. 2428 (Ws).

VERBENA THYMOIDES Cham.

Additional synonymy: Verbena thymoides L. ex Bocq., Adansonia 3: 203, sphalm. 1863.

Additional bibliography: Bocq., Adansonia 3: [Rev. Verbenac.] 203. 1863; Moldenke, Phytologia 13: 274. 1966; Moldenke, Résumé Suppl. 14: 10. 1966'.

VERBENA TRIFIDA H.S.K.

Additional bibliography: Bocq., Adansonia 3: [Rev. Verbenac.] 203. 1863: Moldenke, Phytologia il: 318--320. 1965.

VERBENA URTICIFOLIA I.

Additional bibliography: Bocq., Adansonia 3: [Rev. Verbenac.] 203. 1863; W. Stone, Ann. Rep. N. J. State Mus. 1910: 660. 1911; C. Robertson, Psyche 29: 159-173. 1922; H. C. Greene, Trans . Wisc. Acad. Sci. 42: 70. 1953; E. W. Lathrop, Univ. Kans. Sci. Bull. 39: 135 \& 173. 1958; H. C. Greene, Trans. Wisc. Acad. Sci. 53: 194-195. 1964; Percival, Flor. Biol. 131. 1965; R. B. Clarkson, Castanea 31: 100. 1966; Rickett, Wild Fls. U. S. I (2): 362364, pl. 113. 1966; Moldenke, Phytologia 13: 274-275. 1966.

Additional illustrations: Rickett, Wild Fls. U. S. I (2): pl. 113 [in color]. 1966.

Robertson (1922) and Percival (1965) report that the oligolectic bee, Verbenajus verbenae, a member of the family Panurgidae, collects only from this species, V. hastata L., and V. stricta Vent. "The flowers have the anthers included in the narrow-tubed corolla; and the bee extracts the pollen by inserting its front tarsi into the tube and drawing it up with its curled spines".

Greene (1964) reports that this plant is host to the fungus, Ascochyta cunecmaculata Greene. In Kansas, Lathrop (1958) telis us to look for V. urticifolia in the understory of floodplain woods, where it is prominent in autumn, in creek valley thickets, low prairle pastures, and waste places.

The T. Drummond s.n. [New Orleans, 1833], distributed as V. urticifolia, is actually V. scabra Vahl.

Additional citations: $\overline{O H} \bar{O}$ : Dearbourne Co.: A. R. Holdenke 1245 (Rf). Fairfield Co.: A. R. Moldenke 1237 (Rf). Highland Co.: A. R. Moldenke 12Ll (AC). Lawrence Co.: A. R. Moldenke 1251

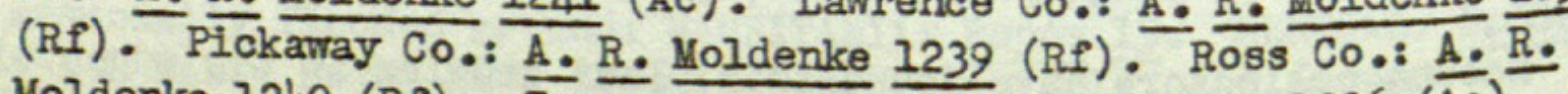

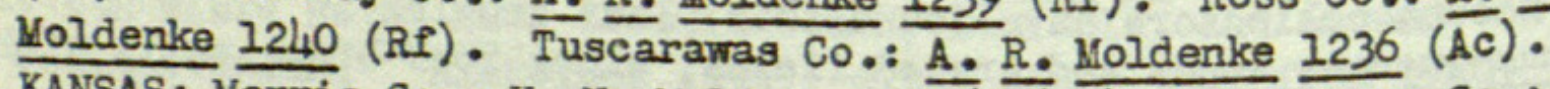
KANSAS: Morris Co.: H. N. Moldenke 23152 (Rf). Wabaunsee Co.:

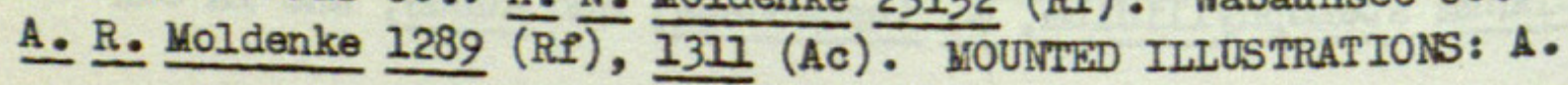




\section{$2 \mathrm{BHL}$ Biodiversity Heritage Library}

Moldenke, Harold N. 1967. "Additional notes on the genus Verbena. III." Phytologia 14, 275-301. https://doi.org/10.5962/bhl.part.18527.

View This Item Online: https://www.biodiversitylibrary.org/item/51598

DOI: https://doi.org/10.5962/bhl.part.18527

Permalink: https://www.biodiversitylibrary.org/partpdf/18527

\section{Holding Institution}

Missouri Botanical Garden, Peter H. Raven Library

\section{Sponsored by}

Missouri Botanical Garden

\section{Copyright \& Reuse}

Copyright Status: In copyright. Digitized with the permission of the rights holder.

Rights Holder: Phytologia

License: http://creativecommons.org/licenses/by-nc-sa/3.0/

Rights: https://biodiversitylibrary.org/permissions

This document was created from content at the Biodiversity Heritage Library, the world's largest open access digital library for biodiversity literature and archives. Visit BHL at https://www.biodiversitylibrary.org. 